УДК 661.666.1

ИЗМЕНЕНИЕ ТЕКСТУРНЫХ ХАРАКТЕРИСТИК

АКТИВИРОВАННЫХ УГЛЕЙ МЕТОДОМ СУЖЕНИЯ ПОР

ПИРОУГЛЕРОДОМ

\title{
MODIFICATION TEXTURAL CHARACTERISTICS OF ACTIVATED CARBONS BY THE CARBON VAPOR DEPOSITION
}

\begin{abstract}
А.Е. Семёнов
Уфимский государственный нефтяной технический университет, г. Уфа, Российская Федерация

Б.С. Жирнов

Уфимский государственный нефтяной технический университет, филиал, г. Салават, Российская Федерация

Albert E. Semyonov
\end{abstract}

Ufa State Petroleum Technological University, Ufa, Russian Federation Boris S. Zhirnov

Ufa State Petroleum Technological University, Branch, Salavat, Russian Federation

e-mail: semenv-albert@rambler.ru

Аннотация. В настоящей работе приведено исследование процесса изменения текстурных характеристик (сужение пор адсорбентов) методом отложения пироуглерода из паровой фазы на поверхности различных углеродных материалов: промышленные активированные угли и образцы, изготовленные в лабораторных условиях. Полученные в лабораторных условиях образцы (СПЩ800) изготавливались путём смешения сажи с 
нефтяным пеком с дальнейшей их карбонизацией, щелочной активацией и последовательной промывкой раствором соляной кислоты и дистиллированной водой от непрореагировавшей щелочи после активации. Процесс сужения пор вели путём пиролиза паров бензола в токе инертного газа через нагретую до постоянной температуры навеску активного угля в течение от 1 до 8 ч. Полученные модифицированные образцы исследовали методом молекулярных щупов, для чего измеряли изотермы адсорбции бензола и четырёххлористого углерода на одних и тех же образцах. Из данных изотерм были рассчитаны удельная поверхность и объём микропор соответственно. Сравнивая изотермы адсорбции по различным адсорбтивам, оценили долю микропор шириной 0,4-0,6 нм, что может косвенно судить об однородности распределения пор по размерам. Результаты исследований показали, что с увеличением времени процесса сужения пор пироуглеродом от 1 до 8 ч величина привеса увеличивается до 15 \%. Наряду с этим происходит также увеличение удельного объёма микропор шириной от 0,4-0,6 нм до величины 0,07-0,15 cм³/г в зависимости от исходных текстурных характеристик адсорбентов. Данные результаты свидетельствуют о том, что полученные таким образом модифицированные активные угли могут быть использованы для получения исходного сырья, промышленных углеродных молекулярных сит с регулируемыми параметрами (ширина пор и иные текстурные характеристики), которые необходимы для разделения заданных смесей.

Abstract. In this study the process of changing the textural characteristics (modification of the pores sizes of adsorbents) by the method of deposition of pyrocarbon from the vapor phase on the surface of various carbon materials: industrial activated carbons and samples prepared in laboratory conditions. The laboratory samples (SPSH800) were made by mixing carbon black with petroleum pitch with their further carbonization, alkaline activation and sequential washing with a solution of hydrochloric acid and distilled water from unreacted alkali after activation. The process of pore narrowing was carried out by pyrolysis 
of benzene vapor in an inert gas stream through a sample of active carbon heated to a constant temperature for 1 hour to 8 hours. The resulting modified samples were investigated by the molecular probe method, for which the adsorption isotherms of benzene and carbon tetrachloride were measured on the same samples. From these isotherms, the specific surface area and volume of micropores were calculated, respectively. Comparing the adsorption isotherms for various adsorptives, the fraction of micropores with a width of 0.4-0.6 nm was estimated, which can indirectly judge the uniformity of the pore size distribution. The research results showed that with an increase in the time of the process of narrowing the pores by pyrocarbon from 1 hour to 8 hours, the weight gain increases to $15 \%$. Along with this, there is also an increase in the specific volume of micropores with a width from $0.4-0.6 \mathrm{~nm}$ to a value of $0.07-0.15 \mathrm{~cm}^{3} / \mathrm{g}$, depending on the initial textural characteristics of the adsorbents. These results indicate that the modified activated carbons obtained in this way can be used to obtain feedstock for the production of industrial carbon molecular sieves with adjustable parameters (pore width and other textural characteristics), which are necessary for separating specified mixtures.

Ключевые слова: пироуглерод; модифицированный уголь; бензол; адсорбенты; активация; удельная поверхность; адсорбция; углеродные молекулярные сита

Key words: pyrocarbon; modificated carbon; benzene; adsorbents; activation; specific surface area; adsorption; carbon molecular sieves

Из литературы известно, что особым типом активных углей являются углеродные молекулярные сита (УМС), которые используются в основном для выделения азота из воздуха [1]. Данный процесс протекает по так называемому кинетическому механизму: кинетический диаметр молекул азота и кислорода хоть и незначительно, но всё же отличается $(0,364$ и 0,346 нм соответственно). Применяемые для этой цели УМС должны иметь 
микропоры шириной порядка 0,3-0,4 нм. Заданную текстурную характеристику можно осуществить следующими способами:

- термическое разложение полимеров [2, 3];

- компаундирование каменного угля и каменноугольной смолы с дальнейшей карбонизацией [4];

- контролируемая активация физическими и (или) химическими агентами с последующей карбонизацией [5];

- отложение пироуглерода из паровой фазы на поверхности активного угля $[6,7]$.

Приведённые выше исследования показали, что наиболее эффективным методом является отложение пироуглерода из паровой фазы на поверхности активных углей вследствие возможности регулирования ширины пор путём подбора параметров ведения процесса (температура, время пребывания, концентрация, режим движения). В данном способе получения УМС путём отложения пироуглерода из паровой фазы весьма важно, чтобы исходный активированный уголь имел однородное распределение пор. Рекомендуемая ширина пор составляет от 0,4 до 0,6 нм. Только при достижении однородности текстурных характеристик могут быть получены адсорбенты с требуемым сочетанием высокой адсорбционной ёмкости по разделяемым газам и селективности [7].

К сожалению, многие активные угли, в особенности те, которые были получены путём паровой активации (например АГ-3), не отвечают приведённым выше требованиям: ширина их пор зачастую составляет от 0,4 до 2,0 нм, т.е. превышает рекомендованные значения. К тому же, неоднородное распределение по размерам микропор и субмикропор довольно широкое, что может привести к неизбежной блокировке полезной части объёма микропор.

В соответствии с работой [7] следует, что процесс отложения пироуглерода из паровой фазы наиболее эффективно проводить во 
внутреннем диффузионном режиме, так как в этом режиме пироуглерод откладывается главным образом у входа поры [8].

Чтобы достичь заданный режим сужения пор, данный процесс рекомендуется проводить при температурах от $700{ }^{\circ} \mathrm{C}$ до $900{ }^{\circ} \mathrm{C}$. Очевидно, что процесс протекания реакции отложения пироуглерода будет зависеть главным образом от температуры реакции и, следовательно, для исходного угля, имеющего изначально более широкие микропоры (например более 0,6 нм), рекомендуется проводить процесс при более высоких температурах (800 ${ }^{\circ} \mathrm{C}$ и более). Данной методикой можно сузить микропоры и на основе полученного материала проводить процесс дальше уже при более низких температурах с целью получением УМС для разделения газовых смесей.

Целью настоящей работы является исследование эффекта сужения пор методом отложения пироуглерода из газовой фазы на промышленных адсорбентах и образцах, полученных в лабораторных условиях, имеющих различную начальную текстурную характеристику.

\section{Экспериментальная часть}

Для получения образцов использовали как промышленные активные угли СКТ и АГ-3, полученные паровой активацией, так и образец активного угля, приготовленного из сажи и нефтяного пека с помощью щелочной активации. Все исходные образцы представляли собой экструдаты с диаметром около 2 мм и длиной 2-5 мм.

Согласно использованной методике [9] для получения гранул лабораторного образца сажу и пек смешивали в соотношении 50 : 50 и добавляли гидроксид калия в количестве 100 \% в расчёте на сухую смесь сажи и пека. После добавления растворителя полученную смесь гранулировали, гранулы карбонизовали при $800{ }^{\circ} \mathrm{C}$ в атмосфере азота; после охлаждения гранулы многократно промывали раствором соляной кислоты и дистиллированной воды, далее следовала стадия сушки. 
Процедура сужения пор исходного активного угля проводилась по схеме, представленной на рисунке 1.

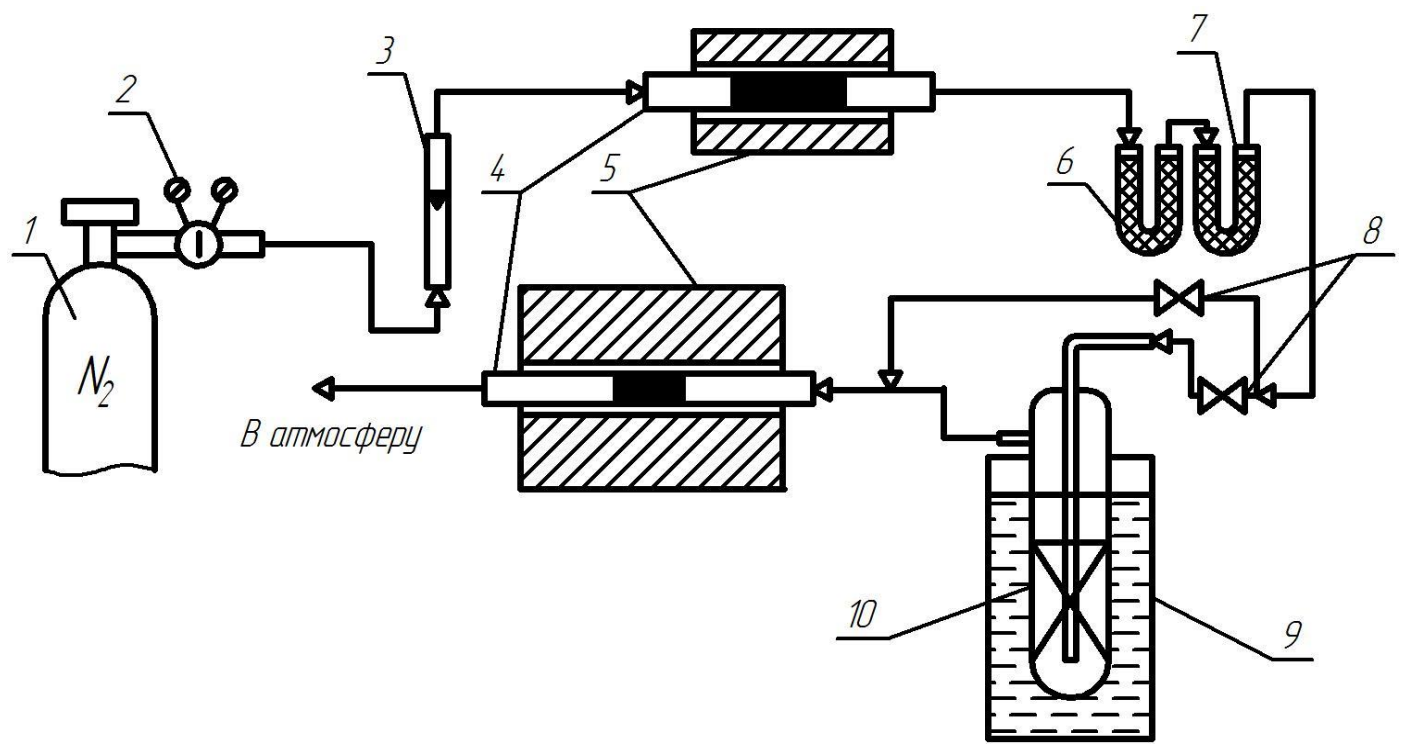

1 - баллон с азотом; 2 - редуктор; 3 - ротаметр;

4 - кварцевые трубки; 5 - электропечи;

6 - U-образная трубка с цеолитом;

7 - U-образная трубка с аскаритом; 8 - зажимы;

9 - термостат; 10 - барботёр с бензолом

Рисунок 1. Схема лабораторной установки получения УМС

Технический азот через редуктор 2 и расходомер 3 поступает в предварительно нагретую до $700{ }^{\circ} \mathrm{C}$ электрическую печь 5, в кварцевой трубке 4 которой находится активированный уголь. При высокой температуре кислород, находящийся в токе технического азота, реагирует с раскалённым активированным углём, в результате чего образуется углекислый газ, который в отличие от кислорода является слабым активирующим агентом. Пройдя через слой активированного угля, очищенный от кислорода азот поступает на дальнейшую подготовку в адсорберы 6 и 7.

В адсорбере 6 происходит осушение азота от оставшейся влаги цеолитом. В адсорбере 7 газ очищается слоем аскарита от углекислого газа, выделившегося в результате термолиза активированного угля и кислорода. Азот, прошедший все предусмотренные стадии очистки, направляется в 
реакционную электрическую печь 5, в кварцевой трубке 4 которой находится предварительно высушенная до постоянной массы и взвешенная проба образца. В данной печи образец угля в токе азота нагревается до температуры 750-900 C. Температура в печи регулируется при помощи автоматического контроллера, входной сигнал на который поступает от термопары типа ТХК; регулирующее воздействие от контроллера поступает на ЛАТР.

После достижения заданной температуры открывается зажим 8 на линии подачи азота в барботёр с бензолом 10, а зажим, установленный на линии продувки печи, закрывается. С целью поддержания заданной концентрации паров бензола на входе в реактор температура в барботёре поддерживается постоянной и составляет $20{ }^{\circ} \mathrm{C}$. В результате высокотемпературного разложения бензола происходит процесс сужения пор на поверхности активированного угля.

После завершения опыта открывается зажим на линии продувки печи, а зажим, установленный на линии подачи в барботёр, - закрывается. Далее полученный образец охлаждается в токе азота до комнатной температуры.

Выгруженный из реактора образец высушивали при $120{ }^{\circ} \mathrm{C}$ до постоянной массы и определяли величину привеса, которую выражали в процентах с учётом поправки потери массы холостого образца, прокаленного в токе азота без подачи бензола в течение 2 ч.

Для всех полученных образцов, в том числе и для исходных, измеряли изотермы адсорбции бензола и четырёххлористого углерода динамическим методом при $20^{\circ} \mathrm{C}[10]$.

Путем обработки изотерм с помощью уравнения БЭТ [11] рассчитывали удельную поверхность образцов. Для определения объема микропор применяли $\alpha_{S}$-метод, предложенный Сингом [11], который заключается в сравнении изотерм адсорбции для исследуемого и стандартного образцов. В качестве стандарта в настоящей работе использовали данные по 
адсорбции бензола [12] и четырёххлористого углерода [13] на графитированной саже.

\section{Результаты и обсуждение}

Текстурные характеристики исходных образцов представлены в таблице 1.

Таблица 1. Характеристики исходных активных углей

\begin{tabular}{|l|c|c|c|c|c|}
\hline \multirow{2}{*}{ Образец } & \multicolumn{2}{|c|}{ Изотерма $\mathrm{C}_{6} \mathrm{H}_{6}$} & \multicolumn{2}{c|}{ Изотерма $\mathrm{CCl}_{4}$} & $\Delta \mathrm{V}_{\text {микро }}$ \\
\cline { 2 - 5 } & $\mathrm{S}_{\text {Бэт }}, \mathrm{M}^{2} / \Gamma$ & $\mathrm{V}_{\text {микро }}, \mathrm{cm}^{3} / \Gamma$ & $\mathrm{S}_{\text {Бэт, }} \mathrm{M}^{2} / \Gamma$ & $\mathrm{V}_{\text {микро }}, \mathrm{cm}^{3} / \Gamma$ & $\mathrm{cm}^{3} / \Gamma$ \\
\hline СКТ & 890 & 0,413 & 694 & 0,351 & 0,062 \\
\hline СПЩ800 & 423 & 0,213 & 380 & 0,203 & 0,010 \\
\hline АГ-3 & 796 & 0,404 & 754 & 0,393 & 0,011 \\
\hline
\end{tabular}

Угли АГ-3 и СКТ, как можно заметить из таблицы 1, имеют высокие значения площади удельной поверхности и объём микропор в отличие от угля, который был приготовлен в лабораторных условиях. Данное различие обосновывается тем, что промышленные угли относятся к газовому типу, так как предназначены для тонкой очистки газов и воды, что соответствует углям с развитой поверхностью микропор [14]. Однако данная характеристика лишь косвенно отражает текстуру исследуемых микропористых материалов [15]. Поэтому более важным показателем является объём микропор, доступных для конкретного адсорбтива. В данной работе использовали метод молекулярных щупов.

Из литературы известно, что молекулы бензола и четырёххлористого углерода адсорбируются соответственно в микропорах шириной 0,4 и 0,6 нм и более $[11,15,16]$. То есть разность данных величин, представленная в последнем столбце, может служить для оценки объёма микропор, размер которых лежит в пределах 0,4-0,6 нм. Поэтому по таблице 1 можно сделать вывод, что наиболее значимым объёмом таких микропор обладает лишь уголь СКТ. Наглядно этот факт демонстрируется на графике (рисунок 2), на котором представлены изотермы адсорбции разных адсорбтивов для одного 
и того же образца, причём количество поглощённого вещества выражено в объёмах жидкого адсорбтива при температуре опыта. Для активных углей АГ-3 и СПЩ800 эти изотермы практически сливаются, в то время как для угля СКТ изотерма для четырёххлористого углерода располагается заметно ниже, чем для бензола (рисунок 2).

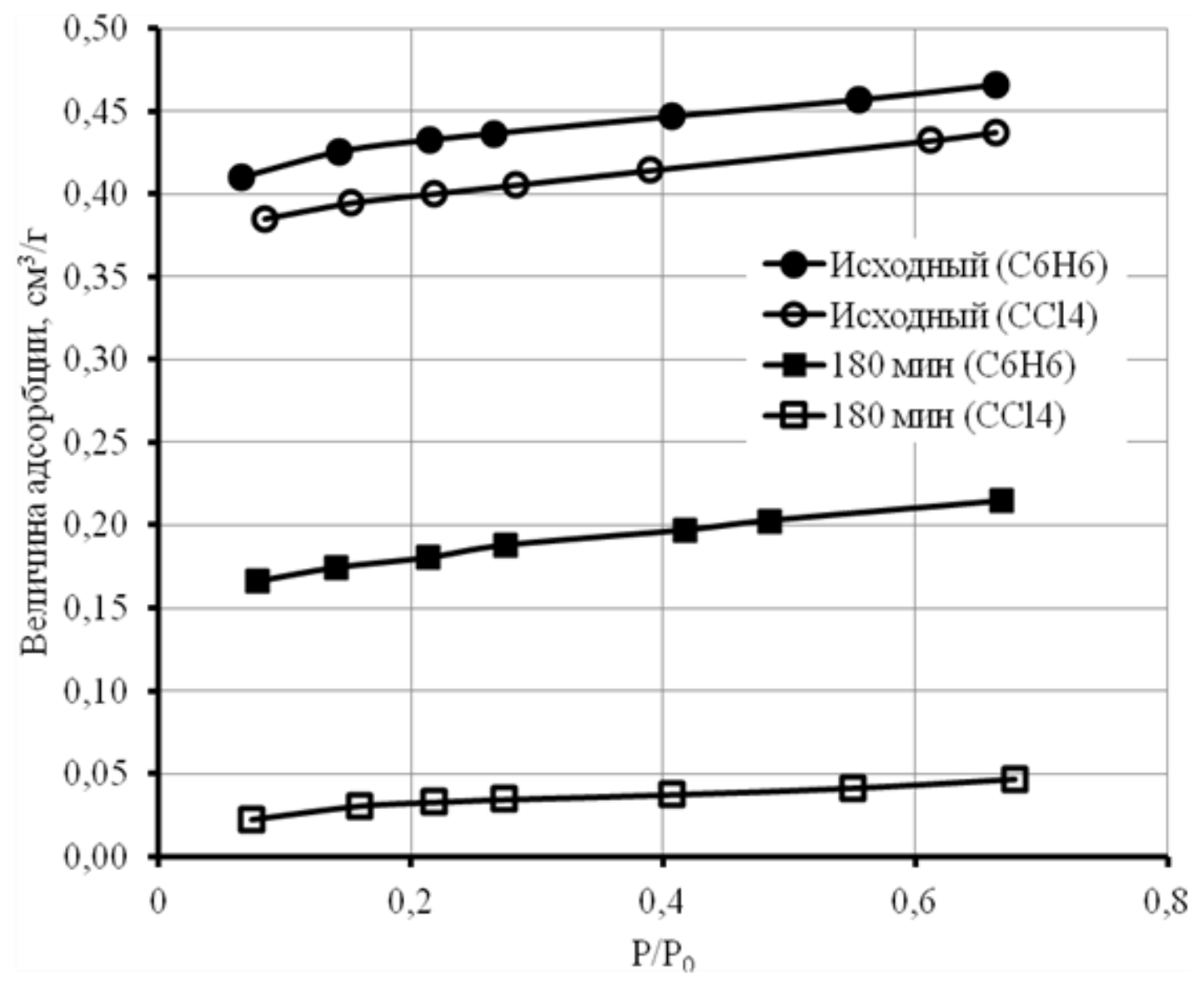

Рисунок 2. Изотермы адсорбции бензола и четырёххлористого углерода на исходном и модифицированном угле СКТ

В ходе экспериментов время проведения процесса сужения пор изменяли в пределах 60-450 мин в зависимости от образца. При этом с увеличением времени наблюдался рост величины привеса. В качестве примера на рисунке 3 приведён график зависимости привеса от времени проведения опыта для угля СКТ и АГ-3. 


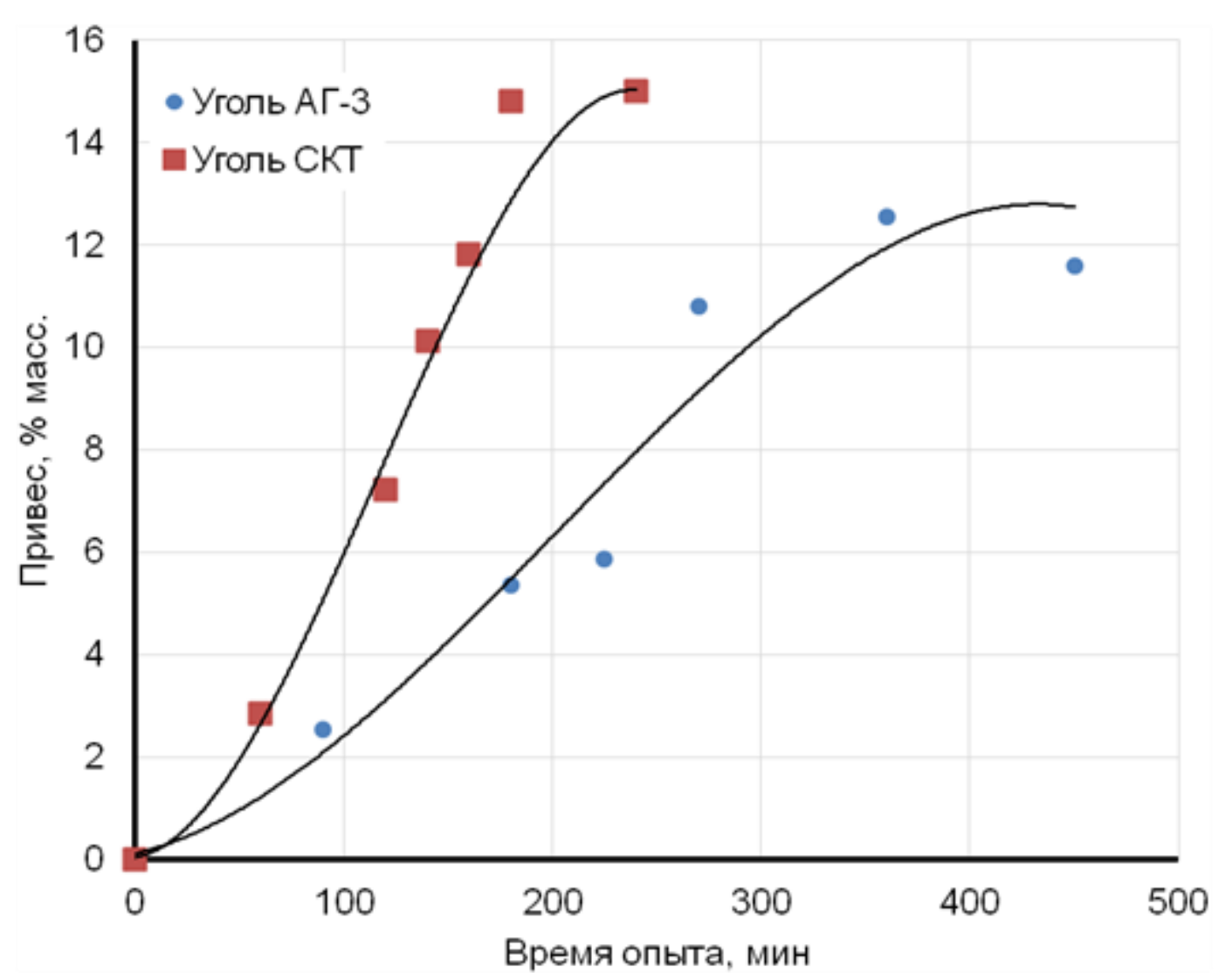

Рисунок 3. Зависимость количества привеса от времени проведения опыта для активного угля СКТ и АГ-3

Рисунок 3 показывает, что по форме полученные зависимости напоминают S-образную кривую, что можно объяснить следующим образом:

- на начальном участке скорость отложения незначительна, повидимому, из-за плохого сродства образующихся слоев пироуглерода к поверхности активного угля, которая может быть образована как базисными плоскостями, так и выходящими на поверхность торцами данных плоскостей [17];

- в средней части кривых можно наблюдать прямолинейные участки, наличие которых может быть связано с установившимся режимом отложения пироуглерода на поверхности угля; 
- при дальнейшем увеличении времени эксперимента можно наблюдать снижение скорости отложения, обусловленное закрытием пор и тем самым, сокращением доступной поверхности.

Аналогичное поведение прослеживается и у активных углей СПЩ800.

Очевидно, что с точки зрения получения углеродных молекулярных сит интерес представляет средний участок, на котором сужение должно уже быть существенно, но при этом не происходит полной блокировки пор.

На рисунке 2 представлены изотермы адсорбции четырёххлористого углерода и бензола для исходного угля СКТ и образца, полученного на его основе в результате сужения пор пироуглеродом в течение 180 мин. Из рисунка 2 видно, что для обоих адсорбтивов изотермы располагаются значительно ниже исходных, что указывает на эффект частичной блокировки пор. С другой стороны, увеличивается расстояние между соответствующими изотермами, что свидетельствует об эффекте сужения пор и увеличении объёма микропор с размером 0,4-0,6 нм.

В таблице 2 показаны результаты обработки изотерм адсорбции бензола и четырёххлористого углерода для образцов, полученных при времени процесса, соответствующего прямолинейному участку подъёма в средней части кривой зависимости привеса от времени для каждого типа активного угля.

Таблица 2 показывает, что величина привеса для различных образцов изменяется в пределах от 3 до 15 \%, причём «плато» (конечный участок кривой зависимости привеса от времени) находится примерно на одном уровне для различных типов углей: около 10-15 \%. Хорошо заметно, что с увеличением времени сужения пор снижаются и удельная поверхность, и объём микропор для всех образцов. При этом также увеличивается объём микропор $\left(\Delta \mathrm{V}_{\text {микро }}\right)$, размер которых лежит в пределах $0,4-0,6$ нм. Из таблицы 2 видно, что наибольшего значения данная величина достигает для угля СКТ, наименьшего - для угля АГ-3; приготовленный нами образец на 
основе сажи и нефтяного пека с добавкой щелочи занимает промежуточное положение.

Таблица 2. Характеристики модифицированных углей

\begin{tabular}{|c|c|c|c|c|c|c|c|c|}
\hline \multirow[b]{2}{*}{ Образец } & \multirow{2}{*}{$\begin{array}{c}\text { Время } \\
\text { процесса, } \\
\text { мин }\end{array}$} & \multirow[b]{2}{*}{ Привес, \% } & \multicolumn{2}{|c|}{ Бензол } & \multicolumn{2}{|c|}{$\mathrm{CCl}_{4}$} & \multirow[b]{2}{*}{$\begin{array}{c}\Delta \mathrm{V}_{\text {микро, }}, \\
\mathrm{cm}^{3} / \Gamma\end{array}$} & \multirow{2}{*}{$\begin{array}{c}\text { Доля } \\
\text { целевых } \\
\text { микропор } \\
\%\end{array}$} \\
\hline & & & $\begin{array}{l}\mathrm{S}_{\text {БэТ }}, \\
\mathrm{M}^{2} / \Gamma\end{array}$ & $\begin{array}{l}\mathrm{V}_{\text {микро, }} \\
\mathrm{cm}^{3} / \Gamma\end{array}$ & $\begin{array}{l}\mathrm{S}_{\text {БЭТ }}, \\
\mathrm{M}^{2} / \Gamma\end{array}$ & $\begin{array}{l}\mathrm{V}_{\text {микро }}, \\
\mathrm{cm}^{3} / \Gamma\end{array}$ & & \\
\hline \multirow{3}{*}{ CKT } & 140 & 10,13 & 698 & 0,357 & 438 & 0,214 & 0,143 & 34,6 \\
\hline & 160 & 11,81 & 615 & 0,297 & 320 & 0,150 & 0,147 & 35,6 \\
\hline & 180 & 14,81 & 358 & 0,168 & 67 & 0,015 & 0,153 & 37,1 \\
\hline \multirow{3}{*}{ СПЩ800 } & 120 & 3,47 & 398 & 0,203 & 348 & 0,182 & 0,021 & 9,7 \\
\hline & 180 & 6,74 & 371 & 0,189 & 243 & 0,124 & 0,065 & 30,3 \\
\hline & 240 & 8,90 & 343 & 0,173 & 203 & 0,101 & 0,072 & 33,8 \\
\hline \multirow{3}{*}{$\mathrm{A} \Gamma-3$} & 180 & 5,37 & 745 & 0,374 & 650 & 0,343 & 0,031 & 7,7 \\
\hline & 270 & 10,80 & 616 & 0,309 & 492 & 0,270 & 0,039 & 9,7 \\
\hline & 360 & 12,54 & 604 & 0,298 & 458 & 0,242 & 0,056 & 13,9 \\
\hline
\end{tabular}

Полученные результаты, по-видимому, можно объяснить различной микропористой структурой исходных активных углей, а точнее распределением объёма микропор по их размерам.

Данные результаты подтверждают рекомендации по получению УМС, приведённые в работе [7], согласно которым исходный активный уголь должен обладать как можно более однородной структурой микропор. К сожалению, данное свойство активных углей относительно плохо поддаётся воспроизводимости в случае их получения методом паровой активацией. С другой стороны, из литературы известно, что активные угли, полученные методом «химической» активации, обладают гораздо более однородной микропористой структурой $[17,18]$. В частности, для полученного нами образца (СПЩ800) преобладающий размер микропор может быть настроен количеством добавленной щелочи и температурой активации на стадии приготовления исходного активного угля так же, как это показано для нефтяных коксов в работе [19]. Подтверждением относительно высокой однородности микропор для полученного активного угля СПЩ800 является относительно высокая доля микропор размером 0,4-0,6 нм в расчёте на 
суммарный исходный объём микропор (33,8 \%), что выше, чем у угля АГ-3, и приближается к образцам, полученным на основе угля СКТ (37 \%).

Поскольку все приготовленные образцы не имеют микропор размером 0,3-0,4 нм, то они не могут быть использованы в качестве УМС для разделения кислорода и азота, поэтому необходимо дальнейшее сужение микропор полученных образцов при ином температурном режиме.

\section{Выводы}

Проведённые исследования по сужению пор активных углей методом отложения пироуглерода из газовой фазы продемонстрировали, что при увеличении времени проведения процесса пиролиза от 1 до 8 ч величина привеса пироуглерода, образованного в результате термического разложения бензола, увеличивается от $3 \%$ до $15 \%$.

Анализ текстурных характеристик методом молекулярных щупов на исследуемых образцах показал, что оптимальное время проведения процесса по величине доли микропор шириной 0,4-0,6 нм может быть выбрано по кривой зависимости количества отложенного пироуглерда от времени проведения опыта, и данная величина составляет от 5 \% до 15 \% в зависимости от текстурных свойств исходного образца.

Анализ показал, что наибольшей долей микропор шириной 0,4-0,6 нм обладают образцы активных углей СКТ, наименьшей - АГ-3. Образец СПЩ800, приготовленный в лабораторных условиях, занимает промежуточное положение по величине микропор. Таким образом, для получения адсорбентов с заданными текстурными характеристиками (доля микропор с заданной шириной) необходим подбор исходного сырья с однородным распределением пор по ширине (как например СКТ или СПЩ800) либо же подбор области ведения процесса сужения пор при других температурах.

Для исходного образца, изготовленного путём смешения сажи и нефтяного пека с последующей щелочной активацией СПЩ800, характерно 
однородное распределение пор по размерам, о чём свидетельствует малая разница между величинами объёма микропор по бензолу и четырёххлористому углероду. Однако после проведения ряда опытов данная величина возросла до $0,072 \mathrm{~cm}^{3} / \Gamma$, что при пересчёте на исходный объём пор составляет долю целевых микропор 33,8 \%. Данная величина близка к активному углю СКТ, имеющего долю целевых микропор 37,1 \%.

Таким образом, результаты анализа текстурных характеристик модифицированных адсорбентов показали, что методом сужения пор активных углей пироуглеродом возможно получение углеродных молекулярных сит с заданными текстурными характеристиками путём подбора температурного режима процесса и исходного сырья.

\section{Список использованной литературы}

1. Gaffney T.R. Porous Solids for Air Separation // Current Opinion in Solid State and Materials Science. 1996. Vol. 1. No. 1. P. 69-75. DOI: 10.1016/S13590286(96)80013-1.

2. Fuertes A.B. Fuertes, Centeno T.A. Carbon Molecular Sieve Membranes from Polyetherimide // Microporous and Mesoporous Mater. 1998. Vol. 26. P. 23-26.

3. Wahby A., Wahby A., Silvestre-Albero J., Sepulveda-Escribano A., Rodriguez-Reinoso F. $\mathrm{CO}_{2}$ Adsorption on Carbon Molecular Sieves // Microporous and Mesoporous Materials. 2012. Vol. 164. P. 280-287. DOI: 10.1016/j.micromeso.2012.06.034.

4. Kouichi M., Junichi H., Kenji H. Production of Molecular Sieving Carbon through Carbonization of Coal Modified by Organic Additives // Carbon. 1991. Vol. 29. Issue 4-5. P. 653-660. DOI: 10.1016/0008-6223(91)90133-4.

5. Toda Y., Yuki N., Toyoda S. Change in Pore Structure of Active Carbon with Heat-Treatment // Carbon. 1972. Vol. 10. Issue 1. P. 13-18. 
6. Juntgen H., Knoblauch K., Harder K. Carbon Molecular Sieves: Production from Coal and Application in Gas Separation. Fuel. 1981. Vol. 60. Issue 9. P. 817-822.

7. Freitas M.M.A., Figueiredo J.L. Preparation of Carbon Molecular Sieves for Gas Separations by Modification of the Pore Sizes of Activated Carbons // Fuel. 2001. Vol. 80. P. 1-6.

8. Cabrera A.L., Zehner J.E., Goe C.G., Gaffney T.R., Farris T.S., Armor J.N. Preparation of Carbon Molecular Sieves, I. Two-Step Hydrocarbon Deposition with a Single Hydrocarbon // Carbon. 1993. Vol. 31. Issue 6. P. 969-976. DOI: 10.1016/0008-6223(93)90200-T.

9. Kugatov P.V., Bashirov I.I., Zhirnov B.S., Akhmetova I.I., Poroshin A.S. Production of Molded Activated Carbon from Carbon Black and Petroleum Pitch by Alkaline Activation // Russian Journal of Applied Chemistry. 2016. Vol. 89. P. 886-890. DOI: 10.1134/S1070427216060069.

10. Рубинштейн А.М., Клячко-Гурвич А.Л. Простые и быстрые методы определения величины поверхности катализаторов // Кинетика и катализ. 1962. T. 3. № 4. C. 599-601.

11. Грег С., Синг К. Адсорбция, удельная поверхность, пористость. М.: Мир, 1984. 306 с.

12. Isirikyan A.A., Kiselev A.V. The Absolute Adsorption Isotherms of Vapors of Nitrogen, Benzene and N-Hexane, and the Heats of Adsorption of Benzene and N-Hexane on Graphitized Carbon Blacks. I. Graphitized Thermal Blacks // Journal of Physical Chemistry. 1961. Vol. 65. Issue 4. P. 601-607. DOI: $10.1021 / \mathrm{j} 100822 \mathrm{a} 004$.

13. Avgul N.N., Kiselev A.V., Lygina I.A., Mikhailova E.A. Isotherms and Heats of Adsorption of Neopentane and Carbon Tetra Chloride Vapor on Graphitized Carbon Black // Bulletin of the Academy of Sciences of the USSR, Division of Chemical Science Volume. 1962. Vol. 11. P. 717-723. DOI: 10.1007/BF00905289. 
14. Колышкин Д.А., Михайлова К.К. Активные угли: Свойства и методы испытаний: справочник. Л.: Химия, 1972. 57 с.

15. Карнаухов А.П. Адсорбция. Текстура дисперсных и пористых материалов. Новосибирск: Наука, 1999. 469 с.

16. Jasienko-Halat M., Kedzior K. Comparison of Molecular Sieve Properties in Microporous Chars from Low-Rank Bituminous Coal Activated by Steam and Carbon Dioxide // Carbon. 2005. Vol. 43. Issue 5. P. 944-953. DOI: 10.1016/j.carbon.2004.11.024.

17. Кинле Х., Бадэр Э. Активные угли и их промышленное применение. Л.: Химия, 1984. 215 с.

18. Marsh H., Rodriguez-Reinoso F. Activated Carbon. London: Elsevier Science and Technology Books, 2006. 536 p.

19. Otowa T., Tanibata R., Itoh M. Production and Adsorption Characteristics of MAXSORB: High-Surface-Area Active Carbon // Gas Separation and Purification. 1993. Vol. 7. No. 4. P. 241-245. DOI: 10.1016/09504214(93)80024-Q.

\section{References}

1. Gaffney T.R. Porous Solids for Air Separation. Current Opinion in Solid State and Materials Science, 1996, Vol. 1, No. 1, pp. 69-75. DOI: 10.1016/S13590286(96)80013-1.

2. Fuertes A.B. Fuertes, Centeno T.A. Carbon Molecular Sieve Membranes from Polyetherimide. Microporous and Mesoporous Mater, 1998, Vol. 26, pp. 2326.

3. Wahby A., Wahby A., Silvestre-Albero J., Sepulveda-Escribano A., Rodriguez-Reinoso F. $\mathrm{CO}_{2}$ Adsorption on Carbon Molecular Sieves. Microporous and Mesoporous Materials, 2012, Vol. 164, pp. 280-287. DOI: 10.1016/j.micromeso.2012.06.034. 
4. Kouichi M., Junichi H., Kenji H. Production of Molecular Sieving Carbon Through Carbonization of Coal Modified by Organic Additives. Carbon, 1991, Vol. 29, Issue 4-5, pp. 653-660. DOI: 10.1016/0008-6223(91)90133-4.

5. Toda Y., Yuki N., Toyoda S. Change in Pore Structure of Active Carbon with Heat-Treatment. Carbon, 1972, Vol. 10, Issue 1, pp. 13-18.

6. Juntgen H., Knoblauch K., Harder K. Carbon Molecular Sieves: Production from Coal and Application in Gas Separation. Fuel, 1981, Vol. 60, Issue 9, pp. 817-822.

7. Freitas M.M.A., Figueiredo J.L. Preparation of Carbon Molecular Sieves for Gas Separations by Modification of the Pore Sizes of Activated Carbons. Fuel, 2001, Vol. 80, pp. 1-6.

8. Cabrera A.L., Zehner J.E., Goe C.G., Gaffney T.R., Farris T.S., Armor J.N. Preparation of Carbon Molecular Sieves, I. Two-Step Hydrocarbon Deposition with a Single Hydrocarbon. Carbon, 1993, Vol. 31, Issue 6, pp. 969-976. DOI: 10.1016/0008-6223(93)90200-T.

9. Kugatov P.V., Bashirov I.I., Zhirnov B.S., Akhmetova I.I., Poroshin A.S. Production of Molded Activated Carbon from Carbon Black and Petroleum Pitch by Alkaline Activation. Russian Journal of Applied Chemistry, 2016, Vol. 89, pp. 886-890. DOI: 10.1134/S1070427216060069.

10. Rubinshtein A.M., Klyachko-Gurvich A.L. Prostye i bystrye metody opredeleniya velichiny poverkhnosti katalizatorov [Simple and Fast Methods for Determining the Size of the Surface of Catalysts]. Kinetika i kataliz - Kinetika $i$ Kataliz, 1962, Vol. 3, No. 4, pp. 599-601. [in Russian].

11. Greg S., Sing K. Adsorbtsiya, udel'naya poverkhnost', poristost' [Adsorption, Specific Surface Area, Porosity]. Moscow, Mir Publ., 1984. 306 p. [in Russian]. 
12. Isirikyan A.A., Kiselev A.V. The Absolute Adsorption Isotherms of Vapors of Nitrogen, Benzene and N-Hexane, and the Heats of Adsorption of Benzene and N-Hexane on Graphitized Carbon Blacks. I. Graphitized Thermal Blacks. Journal of Physical Chemistry, 1961, Vol. 65, Issue 4, pp. 601-607. DOI: $10.1021 / \mathrm{j} 100822 \mathrm{a} 004$.

13. Avgul N.N., Kiselev A.V., Lygina I.A., Mikhailova E.A. Isotherms and Heats of Adsorption of Neopentane and Carbon Tetra Chloride Vapor on Graphitized Carbon Black. Bulletin of the Academy of Sciences of the USSR, Division of Chemical Science Volume, 1962, Vol. 11, pp. 717-723. DOI: 10.1007/BF00905289.

14. Kolyshkin D.A., Mikhailova K.K. Aktivnye ugli: svoistva $i$ metody ispytanii: spravochnik [Activated Carbons: Properties and Test Methods: a Handbook]. Leningrad, Khimiya Publ., 1972. 57 p. [in Russian].

15. Karnaukhov A.P. Adsorbtsiya. Tekstura dispersnykh $i$ poristykh materialov [Adsorption. Dispersed and Porous Textures]. Novosibirsk, Nauka Publ., 1999. 469 p. [in Russian].

16. Jasienko-Halat M., Kedzior K. Comparison of Molecular Sieve Properties in Microporous Chars from Low-Rank Bituminous Coal Activated by Steam and Carbon Dioxide. Carbon, 2005, Vol. 43, Issue 5, pp. 944-953. DOI: 10.1016/j.carbon.2004.11.024.

17. Kinle Kh., Bader E. Aktivnye ugli $i$ ikh promyshlennoe primenenie [Activated Carbons and their Industrial Applications]. Leningrad, Khimiya Publ., 1984. 215 p. [in Russian].

18. Marsh H., Rodriguez-Reinoso F. Activated Carbon. London, Elsevier Science and Technology Books, 2006. 536 p.

19. Otowa T., Tanibata R., Itoh M. Production and Adsorption Characteristics of MAXSORB: High-Surface-Area Active Carbon. Gas Separation and Purification, 1993, Vol. 7, No. 4, pp. 241-245. DOI: 10.1016/0950-4214(93)80024-Q. 


\section{Сведения об авторах}

\section{About the authors}

Семёнов Альберт Евгеньевич, магистрант кафедры «Нефтехимия и химическая технология», УГНТУ, г. Уфа, Российская Федерация

Albert E. Semyonov, Undergraduate Student of Petrochemistry and Chemical Technology Department, USPTU, Ufa, Russian Federation

e-mail: semenv-albert@ rambler.ru

Жирнов Борис Семёнович, д-р техн. наук, профессор кафедры «Химикотехнологические процессы», УГНТУ, филиал, г. Салават, Российская Федерация

Boris S. Zhirnov, Doctor of Engineering Sciences, Professor of Chemical Technological Processes Department, USPTU, Branch, Salavat, Russian Federation

e-mail: jbc2@mail.ru 